\section{Associação entre malária e anemia em área urbana de transmissão do Plasmodium: Mâncio Lima, Acre, Brasil}

\author{
Association between malaria and anemia in an \\ urban area with Plasmodium transmission: \\ Mâncio Lima, Acre State, Brazil
}

\author{
Asociación entre malaria y anemia en un área \\ urbana de transmisión del Plasmodium: Mâncio \\ Lima, Acre, Brasil
}

Eder Ferreira de Arruda 1 Felipe Monteiro de Araujo 1 Maria Gabriela da Silva Guimarães 1 Rudi Nogueira 1 Alanderson Alves Ramalho 1 Monica da Silva-Nunes 1

\title{
Resumo
}

O objetivo deste trabalho é analisar a prevalência de anemia atribuível à malária na população urbana residente no Município de Mâncio Lima, Acre, Brasil. Trata-se de um estudo de coorte não concorrente com 1.167 pessoas acompanhadas por três meses anteriores à data da entrevista mediante dados do SIVEP-Malária. Foram calculadas as medidas de frequência e a prevalência de anemia em pacientes com e sem histórico de malária recente, conforme as variáveis de interesse. Os resultados mostraram que 50,2\% dos indivíduos eram do sexo masculino e 67,96\% encontravam-se na faixa etária de 15 anos ou mais. A prevalência geral de anemia foi de 7,1\%, sendo maior na faixa etária de 6 meses até 5 anos de idade. Com relação ao histórico de malária recente, verificou-se que 8,3\% dos homens que tiveram malária apresentaram anemia. No geral, a prevalência de anemia atribuível à malária foi nulo, exceto para os homens (2,4\%) e no bairro Cobal $(51,4 \%)$. Os resultados demonstram que a prevalência de anemia é baixa e que a contribuição da malária para anemia existe apenas em homens e áreas geográficas específicas.

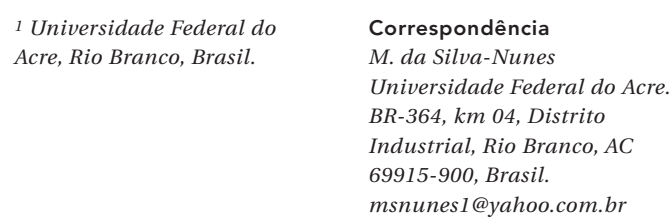

${ }_{1}$ Universidade Federal do Acre, Rio Branco, Brasil.

Correspondência M. da Silva-Nunes Universidade Federal do Acre. BR-364, km 04, Distrito Industrial, Rio Branco, AC 69915-900, Brasil. msnunes1@yahoo.com.br 


\section{Introdução}

Dentre os diversos problemas de saúde pública, a anemia é o distúrbio nutricional mais prevalente em todo o mundo, afetando indivíduos de todos os grupos socioeconômicos e faixas etárias, sobretudo em crianças e mulheres nos países em desenvolvimento 1,2 .

No Acre, Brasil, os estudos desenvolvidos que abordam a anemia como temática descrevem a anemia como um relevante problema de saúde no estado em razão das altas prevalências gerais encontradas 3,4,5,6.

Outro problema de saúde relevante em determinadas áreas do Acre por apresentar elevada incidência é a malária. Nas áreas endêmicas do estado, a malária e a anemia coexistem, mas a relação entre ambas ainda não está bem esclarecida, pois não há estudos que investiguem tal relação.

O presente trabalho teve como objetivo analisar a prevalência de anemia (PA) atribuível à malária na população urbana residente no Município de Mâncio Lima, Acre, para se estabelecer um melhor planejamento de ações de saúde locais e regionais na prevenção e controle de ambas.

\section{Material e método}

O presente trabalho trata-se de um estudo de coorte não concorrente realizado na área urbana do Município Mâncio Lima. Este município está localizado no extremo oeste da região Amazônica e é uma localidade de alta transmissão de malária.

A população geral de Mâncio Lima identificada no Censo Demográfico de 2010 foi de 15.206 habitantes, sendo 7.836 homens e 7.370 mulheres. Cerca de 6.456 pessoas residiam na zona rural e 8.750 na zona urbana ${ }^{7}$. Em janeiro de 2006, o índice parasitário anual (IPA) da área urbana foi maior que 800. Medidas de controle conseguiram reduzir esse índice para cerca de 400, em 2007. Entre 2008 e 2011, o IPA variou entre 100 e 200, conforme a época do ano. Em 2012, ano do nosso estudo, o IPA foi de 74 casos para cada 1.000 habitantes, tendo sido notificados 1.129 casos na área urbana, com $86,6 \%$ por Plasmodium vivax e $13,4 \%$ por Plasmodium falciparum 8 .

Para compor a coorte, foram sorteados $20 \%$ dos domicílios de cada bairro do núcleo urbano do município e todos os indivíduos residentes nesses domicílios foram selecionados para o estudo. A amostragem foi baseada em uma prevalência de $25 \%$ de malária urbana calculada pelos dados de 2010-2011, com 10\% de precisão e uma taxa de perda por não resposta de $10 \%$ (nível de 95\% de confiança).
Assim sendo, foram sorteados, proporcionalmente, 348 domicílios entre os bairros, totalizando uma população de 1.287 indivíduos, correspondendo a $8,47 \%$ da população total e $14,7 \%$ da população urbana. Desses, foram excluídas 32 crianças menores de seis meses. Além disso, houve perda de 88 indivíduos que se recusaram a realizar a coleta sanguínea $(7,01 \%)$, resultando numa população de 1.167 indivíduos que foram acompanhados por um período de três meses, contados retrospectivamente a partir do mês de fevereiro de 2012

Todos os participantes foram entrevistados e tiveram sua concentração de hemoglobina quantificada mediante um hemoglobinômetro portátil (modelo HB 301, HemoCue, Ängelhom, Suécia). Para definição de anemia, adotaram-se os valores preconizados pela Organização Mundial da Saúde (OMS) 1, conforme mostrado na Tabela 1.

Os episódios de malária foram determinados pela consulta de todos os casos de malária registrados no Sistema de Informação de Vigilância Epidemiológica-Malária (SIVEP-Malária) para Mâncio Lima nos três meses anteriores à data da entrevista, de novembro de 2011 a fevereiro de 2012.

A pesquisa foi aprovada pelo Comitê de Ética em Experimentação com Seres Humanos da Universidade Federal do Acre (processo no 23107.016975/2011-28). Todos os indivíduos diagnosticados com anemia e malária na data da entrevista receberam tratamento adequado para essas doenças.

Para a taxa de incidência (TI) de malária recente, calculou-se primeiramente o tempo de acompanhamento dos indivíduos da coorte em pessoas-3meses, somando o tempo de seguimento de cada indivíduo em 3 meses. A TI de malária foi calculada como a razão entre o número de casos de malária notificados como malária recente e o total de pessoa-tempo de contribuição a partir de três meses retrospectivos de acompanhamento, multiplicados por 4 (quatro trimestres em um ano), para se obter o valor em pessoa-ano, segundo a fórmula:

$\mathrm{TI}$ = (número de casos de malária / pessoa-3meses) $\mathrm{x} 4$

A PA foi calculada para cada uma das variáveis investigadas: sexo, faixa etária e bairro de moradia. A idade dos indivíduos foi categorizada de 6 meses a 4 anos e 11 meses, de 5 a 14 anos e 11 meses e maior ou igual a 15 anos. Empregaram-se na análise estatística os testes de quiquadrado de Pearson e exato de Fisher para comparação das prevalências. Foram considerados estatisticamente significantes valores de $\mathrm{p}$ inferiores a 0,05 
Limites de hemoglobina $(\mathrm{Hb})$ utilizados para definir anemia *

\begin{tabular}{lc}
\hline Sexo e/ou grupo etário & Limite de $\mathbf{H b}(\mathbf{g} / \mathbf{d L})$ \\
\hline Crianças (6 meses a 4 anos e 11 meses) & 11,0 \\
Crianças (5 anos a 11 anos e 11 meses) & 11,5 \\
Crianças (12 anos a 14 anos e 11 meses) & 12,0 \\
Mulheres não gestantes ( $\geq 15$ anos) & 12,0 \\
Mulheres gestantes & 11,0 \\
Homens ( $\geq 15$ anos) & 13,0 \\
\hline
\end{tabular}

* Adaptado de Organização Mundial da Saúde 1.

A PA ou excesso de casos prevalentes foi calculada para cada uma das três variáveis investigadas (sexo, faixa etária e bairro de residência) como a diferença entre a PA nos grupos de indivíduos que tiveram malária recente e que não tiveram malária recente, da seguinte forma:

$$
\mathrm{PA}=\mathrm{PA}_{\mathrm{E}}-\mathrm{PA}_{\mathrm{NE}}
$$

Onde $\mathrm{PA}_{\mathrm{E}}$ é a $\mathrm{PA}$ no grupo de pacientes com histórico de malária recente e $\mathrm{PA}_{\mathrm{NE}}$ é a PA no grupo de pacientes que não tiveram malária recente. Intervalos de 95\% de confiança (IC95\%) foram calculados.

\section{Resultados}

Entre os 1.167 indivíduos do estudo, 50,2\% eram do sexo masculino e $49,8 \%$ do sexo feminino; $8,22 \%$ encontravam-se na faixa etária de 6 meses a 4 anos, $23,82 \%$ na faixa de 5 a 14 anos e $67,96 \%$ na faixa etária de 15 anos ou mais. Com relação aos bairros de moradia, 32,6\% das pessoas residiam no bairro São Francisco (Tabela 2).

Há algumas diferenças entre os bairros no que tange a condições de moradia, recebimento de bolsa-família, acesso a esgoto tratado e coleta de lixo, ruas e calçadas pavimentadas, sendo a região central de Mâncio Lima (Bandeirantes, Centro e José Martins) com melhores condições socioeconômicas do que os demais bairros estudados. Já o acesso aos serviços de saúde (consultas de rotina com médico e serviço odontológico) e a disponibilidade de energia elétrica no domicílio foi similar entre os bairros.

Foram notificados 68 casos de malárias (67 por $P$. vivax e 1 por $P$ falciparum) nas 1.167 pessoas, identificadas retrospectivamente pelo SIVEP-Malária nos 90 dias que antecederam a data da entrevista, resultando em uma TI de 0,233 casos de malária para cada pessoa-ano acompanhada, ou 23,3 casos de malárias para cada 100 pessoas acompanhadas por um ano.
No geral, a PA foi de 7,1\%. Para os indivíduos do sexo masculino, a PA foi de $6,2 \%$ e para os indivíduos do sexo feminino foi de $8,1 \%$, não havendo diferença estatisticamente significante (valor de $\mathrm{p}=0,212$ ). Desses, $21,9 \%$ eram crianças na faixa etária de 6 meses a 4 anos, 4,3\% estavam na faixa etária de 5 a 14 anos e 6,3\% na faixa etária de 15 anos ou mais, apresentando diferença estatística (valor de $\mathrm{p}<0,001$ ). Quanto aos bairros de residência, a maior PA foi encontrada no bairro Cobal, porém sem diferença estatística (valor de $\mathrm{p}=0,748$ ), conforme a Tabela 3 .

Com relação à história prévia de malária, verificou-se que $2,6 \%$ das mulheres que tiveram malária nos 90 dias anteriores à entrevista apresentaram anemia. Tal valor aumentou para $8,3 \%$ quando foram avaliados os homens no mesmo período de tempo em relação ao passado malárico, mas sem significância estatística (valor de $\mathrm{p}=0,398$ ).

No que diz respeito ao diagnóstico de anemia nas faixas etárias segundo a história prévia de malária, foi encontrado que $16,7 \%$ das crianças de 6 meses a 4 anos com passado recente de malária tiveram anemia. Já para os indivíduos na faixa etária de 5 a 14 anos de idade, verificou-se que apenas $8,3 \%$ eram anêmicos e tinham histórico de malária prévia, e esse valor decresce para $4,3 \%$ na faixa etária dos indivíduos $\geq 15$ anos de idade, não havendo diferença estatisticamente significante (valor de $\mathrm{p}=0,415$ ). Contudo, a prevalência de anemia nos indivíduos na faixa etária de 6 meses a 4 anos de idade que não tiveram malária nos 90 dias anteriores a entrevista foi de $22,2 \%$, apresentando diferença estatisticamente significante em relação as demais faixas etárias (valor de $\mathrm{p}<0,001$ ).

Para o diagnóstico de anemia nos bairros de moradia de acordo com o passado malárico, $60 \%$ dos indivíduos anêmicos do bairro Cobal tiveram malária nos últimos 90 dias anteriores à entrevista, havendo diferença estatisticamente 
Tabela 2

Características do sexo, faixa etária e distribuição por bairros da população de estudo. Mâncio Lima, Acre, Brasil, 2012.

\begin{tabular}{|c|c|c|c|c|c|c|}
\hline \multirow[t]{2}{*}{ Variáveis } & \multicolumn{2}{|c|}{ População } & \multicolumn{2}{|c|}{ Casos de anemia } & \multicolumn{2}{|c|}{ Pessoas com malária } \\
\hline & $\mathbf{n}$ & $\%$ & $\mathrm{n}$ & $\%$ & $\mathrm{n}$ & $\%$ \\
\hline \multicolumn{7}{|l|}{ Sexo } \\
\hline Masculino & 585 & 50,2 & 36 & 43,4 & 42 & 63,2 \\
\hline Feminino & 582 & 49,8 & 47 & 56,6 & 26 & 36,8 \\
\hline \multicolumn{7}{|l|}{ Faixa etária } \\
\hline 6 meses a 4 anos e 11 meses & 96 & 8,22 & 21 & 25,3 & 12 & 17,6 \\
\hline 5 a 14 anos e 11 meses & 278 & 23,82 & 12 & 14,4 & 36 & 52,9 \\
\hline$\geq 15$ anos & 793 & 67,96 & 50 & 60,3 & 20 & 29,5 \\
\hline \multicolumn{7}{|l|}{ Bairros } \\
\hline Bandeirantes & 103 & 8,8 & 5 & 6,1 & 6 & 8,9 \\
\hline Centro & 154 & 13,2 & 12 & 14,4 & 16 & 23,6 \\
\hline Cobal & 144 & 12,3 & 14 & 16,9 & 30 & 44,1 \\
\hline Iracema & 104 & 8,9 & 9 & 10,8 & 4 & 5,8 \\
\hline José Martins & 48 & 4,1 & 3 & 3,6 & 3 & 4,4 \\
\hline São Francisco & 380 & 32,6 & 23 & 27,7 & 4 & 5,8 \\
\hline São Vidal & 234 & 20,1 & 17 & 20,5 & 5 & 7,4 \\
\hline
\end{tabular}

Tabela 3

Prevalência de anemia (PA), conforme sexo, faixa etária e distribuição por bairros da população de estudo. Mâncio Lima, Acre, Brasil, 2012

\begin{tabular}{|c|c|c|c|}
\hline Variáveis & $\mathbf{n}$ & PA (\%) & Valor de $p$ \\
\hline \multicolumn{4}{|l|}{ Sexo } \\
\hline Masculino & 36 & 6,2 & 0,212 * \\
\hline Feminino & 47 & 8,1 & \\
\hline \multicolumn{4}{|l|}{ Faixa etária } \\
\hline 6 meses a 4 anos e 11 meses & 21 & 21,9 & $<0,001 * *$ \\
\hline 5 a 14 anos e 11 meses & 12 & 4,3 & \\
\hline$\geq 15$ anos & 50 & 6,3 & \\
\hline \multicolumn{4}{|l|}{ Bairros } \\
\hline Bandeirantes & 5 & 4,9 & 0,728 ** \\
\hline Centro & 12 & 7,2 & \\
\hline Cobal & 14 & 10,3 & \\
\hline Iracema & 9 & 7,8 & \\
\hline José Martins & 3 & 5,4 & \\
\hline São Francisco & 23 & 6,4 & \\
\hline São Vidal & 17 & 7,3 & \\
\hline
\end{tabular}

* Teste exato de Fisher;

** Teste de qui-quadrado de Pearson. 
significante entre os demais bairros cuja frequência de anemia foi inferior (valor de $\mathrm{p}<$ 0,001), conforme Tabela 4.

No geral, a PA atribuível à malária na população urbana de Mâncio Lima foi nula. Para os indivíduos do sexo masculino, a prevalência encontrada foi de $2,4 \%$, entretanto para os indivíduos do sexo feminino a PA foi inexistente. Com relação às faixas etárias, os indivíduos na faixa etária de 5 a 14 anos de idade apresentaram uma PA atribuível à malária de 4,3\% e nas demais faixas etárias o valor da PA foi nulo (Tabela 5).

No que diz respeito à PA atribuível a malária nos bairros de moradia, na maioria dos bairros esse valor foi nulo, exceto nos bairros Centro e Cobal nos quais a PA foi de $2,9 \%$ e $51,4 \%$, respectivamente (Tabela 5), significando que a malária pode explicar $51,4 \%$ dos casos de anemia neste bairro do Cobal, e os demais $48,6 \%$ dos casos são devidos a outros fatores que não a malária.

\section{Discussão}

O objetivo do estudo foi identificar se a malária contribui significativamente para a ocorrência de anemia, ou seja, quanto da prevalência de anemia poderia ser justificada pela malária, e, portanto, reduzida caso fossem atingidos níveis ideais de controle da malária. Ao estratificar o excesso de casos prevalentes de anemia atribuível à malária por subgrupos, como idade e sexo, encontrou-se uma pequena proporção de casos de anemia atribuíveis à malária em crianças de 5 anos a 14 anos e 11 meses, e no sexo masculino. Considerando esses resultados, pode-se afirmar que é pequena a contribuição da malária para a ocorrência de anemia na população urbana de Mâncio Lima, sugerindo que existem outros determinantes causais na presente população que contribuem de maneira mais efetiva para o desenvolvimento da anemia.

Dentre as faixas etárias, a maior prevalência de anemia em Mâncio Lima foi registrada em crianças pertencentes à faixa etária de 6 meses a 4 anos sem histórico de malária recente (22,2\%), apresentando diferença estatisticamente significante quando comparada às demais faixas etárias (valor de $\mathrm{p}<0,001$ ). Ainda assim, esse resultado é inferior aos valores encontrados em outros estudos populacionais com crianças nessa mesma faixa etária realizados no próprio Estado do Acre 3,4,5. Isso mostra que nesse grupo etário a malária não contribui significativamente para anemia em Mâncio Lima, pois as crianças estão tendo anemia sem ter tido malária, provavelmente porque a anemia se deve a outras causas. Esse resultado é corroborado por

Tabela 4

Prevalência de anemia em indivíduos com histórico de malária recente e sem histórico de malária recente, conforme sexo, faixa etária e bairro de residência. Mâncio Lima, Acre, Brasil, 2012.

\begin{tabular}{|c|c|c|c|c|}
\hline \multirow[t]{2}{*}{ Variáveis } & \multicolumn{2}{|c|}{ Com histórico de malária recente } & \multicolumn{2}{|c|}{ Sem histórico de malária recente } \\
\hline & $\%$ de anemia & Valor de $\mathrm{p}$ * & $\%$ de anemia & Valor de $p$ * \\
\hline \multicolumn{5}{|l|}{ Sexo } \\
\hline Masculino & 8,3 & 0,398 & 5,9 & 0,124 \\
\hline Feminino & 2,6 & & 8,5 & \\
\hline \multicolumn{5}{|l|}{ Faixa etária } \\
\hline 6 meses a 4 anos e 11 meses & 16,7 & 0,415 & 22,2 & $<0,001$ \\
\hline 5 a 14 anos e 11 meses & 8,3 & & 3,9 & \\
\hline$\geq 15$ anos & 4,3 & & 6,5 & \\
\hline \multicolumn{5}{|l|}{ Bairros } \\
\hline Bandeirantes & 0,0 & $<0,001$ & 5,2 & 0,839 \\
\hline Centro & 10,0 & & 7,0 & \\
\hline Cobal & 60,0 & & 8,5 & \\
\hline Iracema & 0,0 & & 10,4 & \\
\hline José Martins & 0,0 & & 5,9 & \\
\hline São Francisco & 6,1 & & 6,4 & \\
\hline São Vidal & 0,0 & & 7,9 & \\
\hline
\end{tabular}

* Teste de qui-quadrado de Pearson. 
Prevalência de anemia (PA) atribuível à malária, conforme sexo, faixa etária e distribuição por bairros da população de estudo. Mâncio Lima, Acre, Brasil, 2012.

\begin{tabular}{lcc}
\hline Variáveis & PA (\%) & IC95\% \\
\hline Sexo & & 2,4 \\
Masculino & 0,0 & $1,0-5,0$ \\
$\quad$ Feminino & & - \\
Faixa etária & 0,0 & $0,0-7,0$ \\
6 meses a 4 anos e 11 meses & 4,3 & - \\
5 a 14 anos e 11 meses & 0,0 & - \\
$\geq 15$ anos & & $0,0-5,0$ \\
Bairros & 0,0 & $32,0-56,0$ \\
Bandeirantes & 2,9 & - \\
Centro & 51,4 & - \\
Cobal & 0,0 & -
\end{tabular}

IC95\%: intervalo de 95\% de confiança.

Cardoso et al. 9, em estudo sobre malária e anemia no Distrito de Candeias, em Rondônia, onde observaram $37,4 \%$ de anemia em crianças entre 1 e 6 anos sem histórico de malária e 39,8\% de anemia em crianças da mesma faixa etária com histórico de malária recente, diferença essa sem significância estatística.

A PA mais elevada em menores de 5 anos de idade pode ser, teoricamente, explicada por essa fase ser caracterizada por maior velocidade de crescimento, menor frequência e quantidade de consumo de alimentos com fontes de ferro de alta biodisponibilidade. ou ainda por expressiva perda de ferro por causa da espoliação intestinal por enteroparasitos. No caso de crianças expostas à malária, essa perda de ferro pode ocorrer em virtude da hemólise de hemácias pelo Plasmodium 6 .

Com relação ao sexo, a PA em Mâncio Lima foi maior nas mulheres $(8,1 \%)$, embora não tenha havido diferença significativa (valor de $\mathrm{p}=$ 0,212). Esse valor é semelhante ao encontrado em um estudo realizado no Município de Acrelândia $(9,1 \%)$, também no Acre 10 . Por sua vez, difere dos valores constatados em estudos em mulheres não gestantes no Estado de Pernambuco $(21,8 \%) 11$ e no Município de São Leopoldo (21,4\%), no Rio Grande do Sul ${ }^{12}$. Ao analisar a contribuição da malária para anemia separadamente nos sexos masculino e feminino, não houve contribuição nenhuma da malária no sexo feminino, ao passo que para os homens essa medida foi de $2,4 \%$, ou seja, uma pequena porção da anemia encontrada nos homens é decorrente de infecções maláricas recentes, sendo a PA de 8,3\% nos homens e de $2,6 \%$ nas mulheres que tiveram episódios de malária recente (valor de $\mathrm{p}=0,398$ ). Em Porto Velho (Rondônia), um estudo realizado em área de transmissão de Plasmodium, identificou predomínio da incidência de malária e alta frequência de anemia entre os homens, por serem mais expostos em função de suas atividades de trabalho 13 , mas também não identificou nenhuma contribuição da malária para anemia em mulheres.

Portanto, é possível inferir que a anemia identificada entre as mulheres se deve a outros fatores causais e não à malária recente. As principais causas de anemia no sexo feminino estão relacionadas à perda de ferro durante o ciclo menstrual, e em segundo lugar a carência nutricional, segundo dados da literatura 14. Já em homens é pouco frequente a anemia carencial e talvez por essa razão os poucos estudos disponíveis sobre PA em pacientes com malária identifiquem uma parcela de contribuição da malária para os casos de anemia em homens 15 .

Na presente pesquisa, a TI registrada foi de 0,233 casos de malária para cada pessoa-ano que corresponde a 23,3 casos de malárias para cada 100 pessoas acompanhadas por um ano. Esse valor é avaliado como baixo para uma área 
endêmica, e isso pode ser resultado das ações de controle e combate à malária intensificada no estado após o ano de 2006 quando se registrou no Acre sua maior epidemia (93.864 casos). Entre os anos de 2007 e 2008, houve $47 \%$ de redução no número de casos no estado; de 2010 para 2011, houve redução de $37,9 \%$, e quando se compara o ano de 2006 com 2011, verifica-se que ocorreram 75,6\% de redução dos casos de malária no Acre ${ }^{8}$. É possível que a baixa incidência de malária durante o período de estudo tenha diminuído o poder do estudo de quantificar o excesso de casos de anemia atribuível à malária.

Outro fator que pode ter contribuído para a baixa PA atribuível à malária é a reduzida incidência de casos de malária por $P$ falciparum durante o período de estudo. Na África, o risco atribuível populacional da malária por $P$. falciparum para anemia em crianças menores de 5 anos chega a $16,5 \%$, sendo duas vezes maior do que o de parasitoses intestinais 16. Já em Papua Nova Guiné, onde circulam pelo menos três espécies de Plasmodium, 15,1\% da anemia são atribuídos à malária por $P$. falciparum, enquanto outros tipos de malária e infecções mistas contribuem com apenas $12,2 \%$ da malária na população geral avaliada 17 . Embora sejam populações diferentes da população amazônica, com níveis de transmissão de malária mais altos, esses estudos servem para mostrar que a infecção pelo Plasmodium tem o potencial de induzir anemia, conforme o nível de transmissão desse agente infeccioso, e que, portanto, é uma das causas de anemia a ser levada em conta em populações endêmicas, especialmente em situações de epidemia.

Interessantemente, a situação onde a malária pareceu contribuir para um número significativo de casos de anemia foi em algumas áreas específicas da cidade, como o bairro Cobal, onde o excesso de casos de anemia atribuível à malária foi elevado (51,4\%), evidenciando que o bairro de moradia na população de Mâncio Lima é um importante fator na relação entre anemia e malária, pois mais de $50 \%$ da anemia observada nos moradores desse bairro podem ser atribuídas a episódios de malária recente. Dessa forma, a anemia de 5,3\% da população do bairro Cobal (correspondendo a $51,4 \%$ da população anêmica desse bairro) poderia ser eliminada se a malária fosse erradicada da localidade. Essa associação pode ser explicada por diferenças encontradas entre os bairros (Cobal é um dos bairros com população mais desfavorecida em termos socioeconômicos), como trabalho em áreas rurais, banhos em áreas externas do domicílio e casas localizadas em áreas alagadiças. Já o bairro Centro, que possui melhores condições socioeconômicas, teve um pequeno excesso de casos de anemia atribuível à malária (2,9\%), podendo talvez ser o resultado de hábitos que predispõem à malária, como dormir sem mosquiteiro, hábitos de pesca etc. Estudos realizados em outras áreas endêmicas também apontam para diferenças geográficas em pequena escala quanto à contribuição da malária para a anemia, embora não determinem a causa dessa diferença geográfica 17,18 .

Uma importante limitação do estudo é que não foram avaliados valores de hemoglobina antes dos episódios de malária, logo não se pôde detectar a contribuição da malária para variações de níveis de hemoglobina. É possível que o efeito da malária para anemia seja maior do que o mensurado aqui, e, consequentemente, deve ser investigado em estudos futuros sobre o tema.

A PA entre indivíduos com e sem histórico recente de malária permite avaliar o excesso de casos de anemia entre os indivíduos expostos a episódios recentes de malária quando comparados a indivíduos que não tiveram malária. Por conta do fato de a malária ser uma doença aguda e facilmente identificável, permite estudos retrospectivos, já que é uma doença de notificação compulsória. Todavia, a anemia, mesmo sendo temporária como na anemia decorrente da malária, muitas vezes é silenciosa e de difícil detecção. Por causa dessa limitação, pôde-se avaliar somente a PA na população de estudo, não sendo possível medir a incidência de anemia nesse estudo retrospectivo, nem a diferença na incidência de anemia nos indivíduos expostos e não expostos, um dos objetivos iniciais deste estudo. Dessa forma, o cálculo da prevalência atribuível apresenta como principal limitação o fato de não poder mensurar a relação causal entre anemia e malária na população em estudo. No presente trabalho, o excesso de PA em indivíduos com histórico recente de malária se restringiu à determinada faixa etária, sexo e área geográfica do município, sugerindo que mesmo em área endêmica de moderada transmissão, a malária teve pequena associação com anemia nesse tipo de população. Estudos prospectivos poderão investigar melhor a associação entre malária e anemia.

\section{Conclusão}

A malária contribuiu de forma significativa para segmentos específicos da população, como homens com histórico de malária recente (90 dias) e em regiões geográficas definidas, sendo um fator a ser considerado na saúde dessa parte da população. A contribuição da malária para anemia na população urbana de Mâncio Lima possui distribuição espacial bem definida, estando restrita ao 
bairro Cobal, demonstrando assim a necessidade de ações de prevenção e controle da malária mais efetivas em áreas e grupos específicos do município, embora a baixa PA atribuída à malária já seja um indicativo de que as ações nesse sentido estão surtindo efeito.

No presente estudo, as prevalências gerais de anemia mais elevadas foram observadas em crianças e mulheres em idade férteis, reforçando o conhecimento de que tais grupos são os mais vulneráveis. Não houve contribuição significativa da malária para anemia nesses grupos de risco, vale dizer, praticamente nenhum caso de anemia em mulheres e crianças menores de 5 anos pode ser atribuído à malária, ainda que não tenha sido possível avaliar o efeito da malária na redução de níveis de hemoglobina nesses e nos demais grupos populacionais, como já descrito. Há ainda, outros fatores contribuindo para a anemia nesse município, como anemias carenciais em mulheres e crianças menores de 5 anos de idade, que precisam ser avaliadas e controladas pelos serviços de saúde.

\section{Colaboradores}

E. F. Arruda e M. da Silva-Nunes participaram da elaboração e desenho do estudo, da coleta de dados, da análise dos dados e da redação do artigo. F. M. Araujo e A. A. Ramalho participaram do desenho do estudo, da coleta de dados e da análise dos dados; efetuaram revisão crítica do artigo. M. G. S. Guimarães participou do desenho do estudo, da coleta de dados e da análise dos dados de anemia; efetuou revisão crítica do artigo. R. Nogueira participou do desenho do estudo, da coleta de dados e da análise dos dados da SIVEP-Malária; efetuou revisão crítica do artigo.

\section{Agradecimentos}

Os autores agradecem à população de Mâncio Lima, às autoridades de saúde de Mâncio Lima, à Universidade Federal do Acre e ao Programa de Pós-graduação em Saúde Coletiva pelo apoio e colaboração. Os autores agradecem também às agências de financiamento: FDCT/FUNTAC Edital 003/2011, CNPq Edital Universal 2011, FAPAC Edital PPSUS 2013 e UFAC Chamada de Projetos de Pesquisa 2011. 


\section{Referências}

1. World Health Organization. Iron deficiency anaemia: assessment, prevention and control. Geneva: World Health Organization; 2001.

2. Bueno MB, Selem SSC, Arêas JAG, Fisberg RM. Prevalência e fatores associados à anemia entre crianças atendidas em creches públicas de São Paulo. Rev Bras Epidemiol 2006; 9:462-70.

3. Souza OF, Macedo LF, Oliveira CSM, Araujo TS, Muniz PT. Anaemia in children from Rio Branco, AC: prevalence and associated factors. Rev Bras Crescimento Desenvolv Hum 2012; 22:307-13.

4. Castro TG, Silva-Nunes M, Conde WL, Muniz PT, Cardoso MA. Anemia e deficiência de ferro em préescolares da Amazônia Ocidental brasileira: prévalência e fatores associados. Cad Saúde Pública 2011; 27:131-42.

5. Oliveira CC, Cardoso MA, Araújo TS, Muniz PT. Anemia em crianças de 6 a 59 meses e fatores associados no Município de Jordão, Estado do Acre, Brasil. Cad Saúde Pública 2011; 27:1008-20.

6. Melo GC, Reyes-Lecca RC, Vitor-Silva S, Monteiro WM, Martins M, Benzecry SG, et al. Concurrent helminthic infection protects schoolchildren with Plasmodium vivax from anemia. PLoS One 2010; 5:11206.

7. Instituto Brasileiro de Geografia e Estatística. Censo Demográfico, 2010. Rio de Janeiro: Instituto Brasileiro de Geografia e Estatística; 2011.

8. Sistema de Informação de Vigilância Epidemiológica-Malária. Notificação de casos. Relatório. Brasília: Secretária de Vigilância em Saúde, Ministério de Saúde; 2012.

9. Cardoso MA, Ferreira MV, Camargo LMA, Szarfac SC. Anemia em população de área endêmica de malária, Rondônia. Rev Saúde Pública 1992; 26:161-6.

10. Ferreira MU, da Silva-Nunes M, Bertolino CN, Malafronte RS, Muniz PT, Cardoso MA. Anemia and iron deficiency in school children, adolescents, and adults: a community-based study in rural Amazonia. Am J Public Health 2007; 97:237-9.
11. Silva SCL, Batista Filho M, Miglioli TC. Prevalência e fatores de risco de anemia em mães e filhos no Estado de Pernambuco. Rev Bras Epidemiol 2006; 11:266-77.

12. Fabian C, Olinto MTA, Dias-da-Costa JS, Bairros F, Nacul LC. Prevalência de anemia e fatores associados em mulheres adultas residentes em São Leopoldo, Rio Grande do Sul, Brasil. Cad Saúde Pública 2007; 23:1199-205.

13. Katsuragawa TH, Cunha RPA, Souza DCA, Gil LHS, Cruz RB, Silva AA, et al. Malária e aspectos hematológicos em moradores da área de influência dos futuros reservatórios das hidrelétricas de Santo Antônio e Jirau, Rondônia, Brasil. Cad Saúde Pública 2009; 25:1486-92

14. Rodrigues LP, Jorge SRPF. Deficiência de ferro na mulher adulta. Rev Bras Hematol Hemoter 2010; 32:49-52.

15. Cançado RD, Chiattone CS. Anemia ferropênica no adulto - causas, diagnóstico e tratamento. Rev Bras Hematol Hemoter 2010; 32:240-6.

16. Ronald LA, Kenny SL, Klinkenberg E, Akoto AO, Boakye I, Barnish G, et al. Malaria and anaemia among children in two communities of Kumasi, Ghana: a cross-sectional survey. Malar J 2006; 5:1-8.

17. Douglas NM, Lampah DA, Kenangalem E, Simpson JA, Poespoprodjo JR, Sugiarto P, et al. Major burden of severe anemia from non-falciparum malaria species in Southern Papua: a hospital-based surveillance study. PLoS Med 2013; 10:e1001575.

18. Manning L, Laman M, Rosanas-Urgell A, Michon P, Aipit S, Bona C, et al. Severe anemia in Papua New Guinean children from a malaria-endemic area: a case-control etiologic study. PLoS Negl Trop Dis 2012; 6:e1972. 


\section{Abstract}

The aim of this study was to analyze the prevalence of malaria-attributable anemia in the urban population of Mâncio Lima, Acre State, Brazil. This was a non-concurrent cohort study of 1,167 persons followed for the three months prior to the interview using data from the SIVEP-Malaria database. Anemia frequency and prevalence rates were calculated in patients with and without a recent history of malaria, according to target variables. $50.2 \%$ of the individuals were males, and $67.96 \%$ were 15 years or older. Overall anemia prevalence was $7.1 \%$, higher in the 6 months to 5 years age bracket. Some $8.3 \%$ of men with a recent history of malaria presented anemia. Overall, prevalence of malaria-attributable anemia was negligible, except in men (2.4\%) and the Cobal neighborhood (51.4\%). The results showed that anemia prevalence was low and that malaria's contribution to anemia only existed in men and in specific geographic areas.

Malaria; Anemia; Prevalence

\section{Resumen}

El objetivo de este trabajo es analizar la prevalencia de anemia atribuible a la malaria en una población urbana, residente en el Municipio de Mâncio Lima Acre, Brasil. Se trata de un estudio de cohorte no-concurrente con 1.167 personas acompañadas por tre meses anteriores a la fecha de la entrevista a través de datos del SIVEP-Malaria. Se calcularon las medidas de frecuencia y la prevalencia de anemia en pacientes con y sin historial de malaria reciente, conforme las variables de interés. Los resultados mostraron que un 50,2\% de los individuos eran de sexo masculino y 67,96\% se encontraban en la franja de edad de 15 años o más. La prevalencia general de anemia fue de un 7,1\%, siendo mayor en la franja de edad de 6 meses hasta 5 años de edad. En relación con el historial de malaria reciente, se verificó que un 8,3\% de los hombres que tuvieron malaria presentaron anemia. En general, la prevalencia de anemia atribuible a la malaria fue nula, excepto para los hombres $(2,4 \%)$ y en el barrio Cobal (51,4\%). Los resultados demuestran baja prevalencia de anemia y que la contribución de la malaria a la anemia existe solamente en hombres y áreas geográficas específicas.

Malaria; Anemia; Prevalencia
Recebido em 31/Jul/2014

Versão final reapresentada em 05/Dez/2015 Aprovado em 23/Dez/2015 